\title{
SOLID-STATE PULSED POWER MODULE (SSPPM) DESIGN FOR A DENSE PLASMA FOCUS (DPF) DEVICE FOR SEMICONDUCTOR LITHOGRAPHY APPLICATIONS
}

\author{
R.M. Ness and W.N. Partlo \\ CYMER Inc. \\ 16750 Via Del Campo Court \\ San Diego, CA 92127
}

\begin{abstract}
A Dense Plasma Focus (DPF) is being developed at CYMER as a light source for Extreme Ultraviolet (EUV) Lithography applications. The paper describes the Solid State Pulsed Power Module (SSPPM) for driving this device and the application specific requirements for this power system. Based on the same technology used to power CYMER excimer lasers, the SSPPM employs IGBT switching and magnetic pulse compression to generate the pulse delivered to the DPF. The current design generates a $4 \mathrm{kV}, \sim 30 \mathrm{~ns}$ risetime pulse. Two separate charging systems have been used to date. A simple resistive charger allows continuous operation at rep-rates up to $40 \mathrm{~Hz}$ while a resonant charging system currently provides burst mode operation with rep-rates of at least $1 \mathrm{kHz}$. Although relatively small in stored energy compared to many previous DPF machines (the initial capacitor stores $\sim 18 \mathrm{~J}$ ), this system must run at very high rep-rates of at least $5 \mathrm{kHz}$. High rep-rate operation and tight control of the output radiation $(<2 \% 3 \sigma$ pulse-topulse energy repeatability of in-band radiation) are required in order to provide the tight exposure dose control required for semiconductor lithography. This further translates into the requirement for even tighter control and adjustment of the amplitude of the SSPPM output pulse in order to try and maintain a constant radiation output level. Energy recovery is implemented in the SSPPM to recover pulse reflections from the DPF which would normally be dissipated within the DPF and SSPPM as excess heat, lowering the thermal management requirements and reducing the charging system output. Test data from the current generation system are presented along with a discussion of the plans for further evolution of the design towards the final application goals of $5 \mathrm{kHz}$ continuous operation.
\end{abstract}

\section{BACKGROUND AND REQUIREMENTS}

A variety of concepts are being investigated for potential use as an EUV light source, including laser produced plasma sources and discharge sources, such as a dense plasma focus, a Z-pinch, and capillary discharge sources. The general consensus requirements [1] for such a source have been agreed to by the lithography stepper/scanner manufacturers (ASML, Nikon, Canon, and Ultratech Stepper). These requirements, as shown in
Table 1, would support $\sim 80$ wafers/hour ( $300 \mathrm{~mm}$ size) with $5 \mathrm{~mJ} / \mathrm{cm}^{2}$ resist sensitivity and expected mirror reflectivities.

Table 1. EUV Source Requirements.

\begin{tabular}{|c|c|}
\hline Parameter & Requirement \\
\hline $\begin{array}{c}\text { Output Power Captured by } \\
\text { Condenser Optic }\end{array}$ & $50-150 \mathrm{~W}$ in $2 \%$ \\
& $\begin{array}{c}\text { Bandwidth Centered About } \\
13.5 \mathrm{~nm}\end{array}$ \\
\hline Repetition Rate & $\geq 5 \mathrm{kHz}$ \\
\hline Pulse-to-Pulse & $<2 \%(3 \sigma)$ \\
Repeatability & \\
\hline Condenser Lifetime & 1 Year $\left(\sim 1.6 \times 10^{11}\right.$ Pulses $)$ \\
\hline
\end{tabular}

CYMER's approach to an EUV source concept is to utilize a dense plasma focus design based upon similar technology developed for plasma thrusters for space applications [2]. Although a xenon plasma can generate EUV emission, lithium is also being investigated for potential higher conversion efficiencies [3]. A condenser optic (collector) is also being developed based on grazing incidence reflections from multiple concentric shells.

Although dense plasma focus devices have been developed for a number of years, this particular device is somewhat unique in that it is designed to operate at much lower than typical energies and much higher than typical repetition rates. Previous systems were characterized as high rep-rate devices if the DPF was operated at $1-20 \mathrm{~Hz}$ [4] while units with stored energies in the 100's to 1000's of $\mathbf{J}[5]$ were considered low energy experiments. The high rep-rate requirements in this case are necessary in order to average the energy in many pulses for very tight dose control on the wafer. As a result, the SSPPM for this system utilizes both solid-state and magnetic switching to achieve the high rep-rate operation. The system must also allow variable emission output and tight energy control (very tight pulse-to-pulse energy stability) as described in Table 1. A separate pre-ionization system along with the SSPPM design allows the variable output DPF operation with consistent timing and helps to provide a stable emission output. To allow operation with only 10 's of J of electrical energy, the SSPPM also must have an extremely low drive inductance (in the $\mathrm{nH}$ range).

Given the 50-150 W of EUV power requirement and the expectation of an approximate $20 \%$ collection efficiency of the condenser, this implies that $250-750 \mathrm{~W}$ 
of EUV power be generated into $2 \pi$ steradians. At the 5 $\mathrm{kHz}$ rep-rate, this also implies that $50-150 \mathrm{~mJ}$ of EUV energy be generated during each pulse. With the present DPF conversion efficiency of $\sim 0.2 \%$ (comparable to other EUV sources), this requires $125-375 \mathrm{~kW}$ of electrical power be provided to the DPF load. Since the DPF itself is reasonably small in size, cooling such a heat load this size would be difficult. As a result, the implication is that improvements in DPF conversion efficiency are required or further development in the DPF and SSPPM is needed, particularly in the area of thermal management.

\section{DPF SSPPM/POWER SYSTEM DESIGN}

Based on the overall DPF requirements described in Table 1, the DPF SSPPM and Power System design requirements can be extracted and are shown in Table 2 .

Table 2. DPF SSPPM Requirements.

\begin{tabular}{|c|c|}
\hline Parameter & Requirement \\
\hline Input (Charging) Voltage & $\sim 1200-1500 \mathrm{~V}$ \\
\hline Charging Time & $\sim 100-150 \mu \mathrm{s}$ \\
\hline $\begin{array}{c}\text { Charging Regulation } \\
\text { (Pulse-to-Pulse) }\end{array}$ & $< \pm 0.1 \%$ \\
\hline Output Pulse Voltage & $\sim 4-5 \mathrm{kV}$ \\
\hline Output Pulse Risetime & $\sim 30 \mathrm{~ns}$ \\
\hline Output Pulse Energy & $\sim 10-20 \mathrm{~J}$ \\
\hline Repetition Rate & Up to 5 kHz \\
\hline Thermal Management & Direct Water Cooled \\
\hline Other Features & $\begin{array}{c}\text { Energy Recovery to Reduce } \\
\text { Electrode Erosion and } \\
\text { Improve System Efficiency }\end{array}$ \\
\hline
\end{tabular}

As mentioned earlier, a pre-ionization system is also required to support SSPPM and DPF operation. This design utilizes a separate modulator driving 8 spark sources located in a radial array around the DPF cathode. The modulator produces a $\sim 50 \mathrm{kV}, 10 \mathrm{MHz}$ damped sinusoidal pulse to each spark source. The resulting plasma in the DPF provides a finite load impedance to allow saturation of the SSPPM output magnetic switch and helps to ensure consistent DPF operation.

The entire SSPPM design is arranged in a coaxial geometry with an outside diameter of 24" and a length of 26 " as shown in Figure 1. From left to right in the photograph are the magnetic switch bias circuitry deck, the IGBT Trigger and Series Diode deck, the IGBT deck, the $\mathrm{C} 0$ capacitor deck, the $\mathrm{C} 1$ capacitor deck, the Transformer assembly, and the three $\mathrm{C} 2$ capacitor decks. The DPF vacuum vessel connects to the flange on the right end of the assembly with coaxial connections in the center for the DPF anode and cathode.

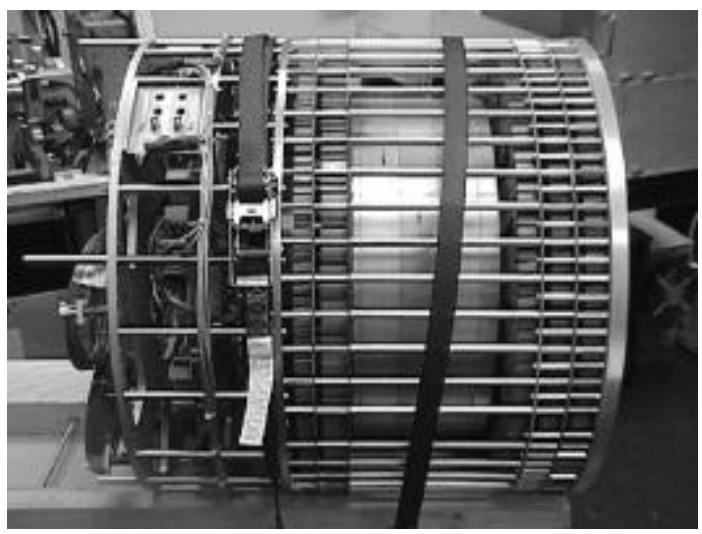

Figure 1. DPF SSPPM Assembly.

A simplified schematic diagram of the DPF SSPPM design is shown in Figure 2. A resonant charger system provides pulse charging of the SSPPM from a $800 \mathrm{Vdc}$, $25 \mathrm{~kW}$ power supply, providing a nominal $1300 \mathrm{~V}$ on the SSPPM initial capacitor store, C0. A de-qing circuit in the resonant charger helps to enable tight regulation of the charging voltage by terminating the charging sequence. The de-qing circuit is also used during the energy recovery process after the main DPF discharge to store reflected energy from the DPF load for use in charging for the next pulse.

The C0 capacitor bank is made up of four "pie" shaped printed circuit board assemblies with $2000 \mathrm{~V}$ polypropylene film capacitors connected in parallel. Eight parallel IGBT switches, S1, transfer the energy stored in C0 through the Series diodes, D1, and a magnetic assist, LS1, into the $\mathrm{C} 1$ capacitor deck, which is similar in construction to the $\mathrm{C} 0$ deck. This initial energy transfer takes $\sim 3-4 \mu$ s until a magnetic switch, LS2, coaxially located within the capacitor deck assemblies then saturates and allows transfer of the energy stored in C1 through a 1:4 step-up transformer and into the three $\mathrm{C} 2$ capacitor decks. This second transfer takes $\sim 500 \mathrm{~ns}$ to complete, at which time the output magnetic switch, LS3, saturates and transfers the energy stored in $\mathrm{C} 2$ into the DPF load.

\section{SSPPM MEASUREMENTS / DATA}

The final transfer of energy into the DPF load results in a voltage pulse applied to the DPF electrodes with a peak of $\sim 4-5 \mathrm{kV}$ and a risetime of $\sim 30 \mathrm{~ns}$. Figure 3 shows this final sequence with the $\mathrm{C} 2$ and Vanode voltages and the EUV emission signal displayed. Although the load current to the DPF is not specifically measured, circuit simulations using the information from these waveforms and known circuit parameters have estimated the load current to be on the order of $\sim 50 \mathrm{kA}$. The total load inductance of the SSPPM and DPF is believed to be $\sim 6$ $\mathrm{nH}$, resulting in a dI/dt at the load of $\sim 700 \mathrm{kA} / \mu \mathrm{s}$. 


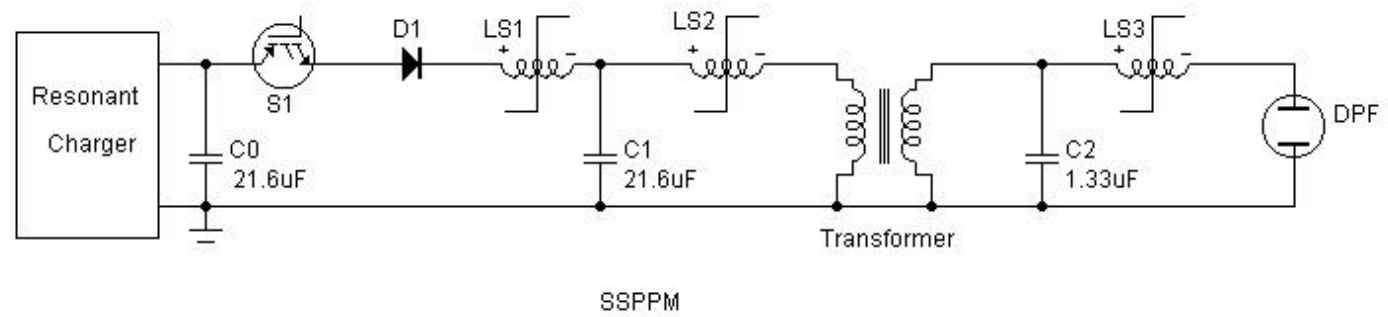

Figure 2. Simplified Schematic Diagram of Dense Plasma Focus (DPF) Solid State Pulsed Power Module (SSPPM).

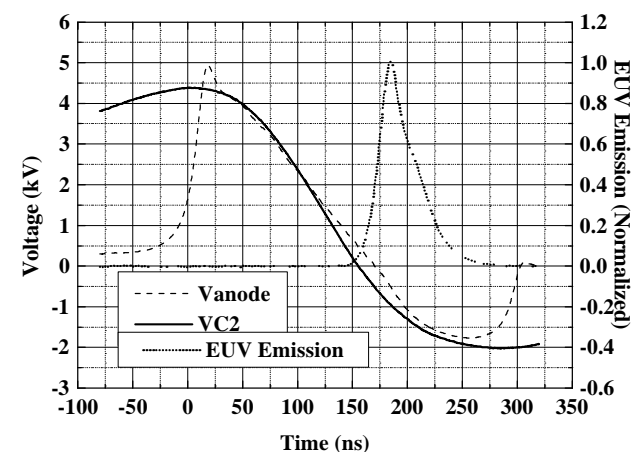

Figure 3. VC2, Vanode, and EUV Emission Signals Showing Output Sequence and Energy Recovery.

One additional item that can be seen from the data in Figure 3 is that both the load and C2 voltages overshoot, resulting in energy that is reflected from the load. This energy is transferred back through the magnetic pulse compression in the SSPPM until it reaches C0 where it too achieves a negative voltage. At this point, the IGBT switches recover and the voltage on $\mathrm{CO}$ is left to freewheel through the charging inductor and a free-wheeling diode in the resonant charger. Since the de-qing switch is still conducting at this point, the energy trapped in the voltage on $\mathrm{C} 0$ is transferred to current in the charging inductor and remains there until the next pulse charging sequence begins. Figure 4 shows the VCO waveform associated with the first two pulses of a $2 \mathrm{kHz}$ burst. From the data, the charging rate on the second pulse is significantly faster than the first pulse due to this recovered energy, dropping from $\sim 234 \mu$ s to $\sim 132 \mu$ s.

Figure 5 shows a second sequence of VC0 waveforms during a $2 \mathrm{kHz}$ burst, this time showing the pulse-to-pulse voltage regulation. As can be seen from the data, with the exception of the first pulse, the variation is less than 6 $\mathrm{Vp}$-p out of $1300 \mathrm{~V}$, or less than $\pm 0.25 \%$. Since the final goal is less than $\pm 0.1 \%$, further improvements to the resonant charger control circuitry are expected in order to improve the regulation control of the de-qing circuit.

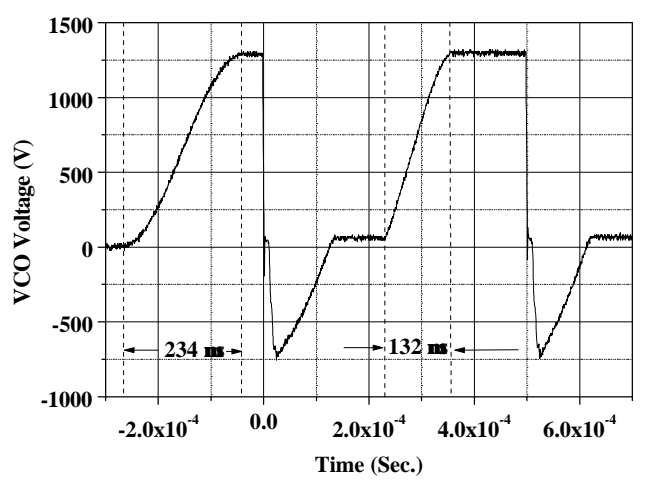

Figure 4. VC0 Waveforms From $2 \mathrm{kHz}$ Burst Demonstrating Energy Recovery.

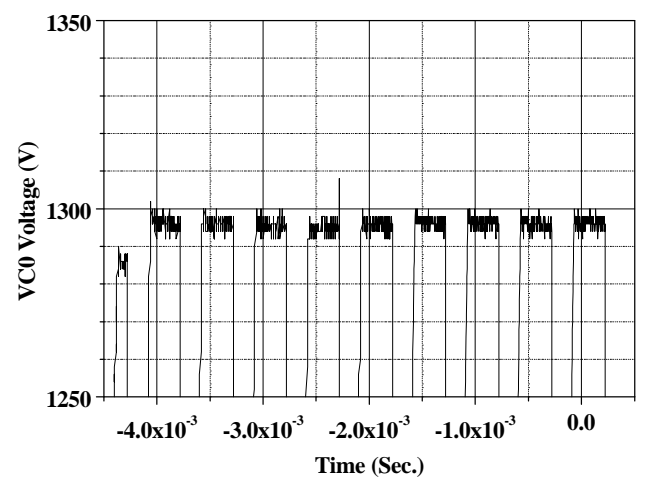

Figure 5. Expanded VC0 Waveforms From $2 \mathrm{kHz}$ Burst Demonstrating Pulse-to-Pulse Voltage Regulation.

\section{EUV EMISSION MEASUREMENTS}

A variety of experiments have been conducted so far with the SSPPM and the DPF system. In-band emission has been measured with load energies as low as $\sim 5 \mathrm{~J}$ using Xenon as the active gas. Both conversion 
efficiency and output energy are approximately linear with input energy from the $5 \mathrm{~J}$ level up to levels as high as $\sim 11 \mathrm{~J}$. Since no fundamental limits have been found yet, additional experiments are planned. As noted earlier, the $\sim 0.2 \%$ efficiency at the high end so far is comparable to other EUV sources in development. EUV emission energy stability (pulse-to-pulse) has also been measured in both continuous and burst mode conditions. When operating in $20 \mathrm{~Hz}$ continuous mode (using simple resistive charging), the DPF exhibits average energies of $\sim 16 \mathrm{~mJ} /$ pulse with a standard deviation $(1 \sigma)$ of $\sim 18 \%$. The corresponding data from a $1 \mathrm{kHz}$ burst, shown in Figure 6 , is $\sim 20 \mathrm{~mJ} /$ pulse and $\sim 16 \%$. As noted in Table 1 , the desired requirement for the EUV source of less than $2 \%(3 \sigma)$ is significantly lower and will therefore require much improvement.

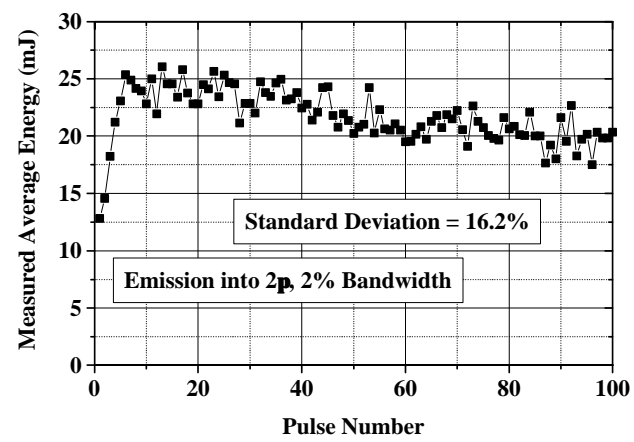

Figure 6. EUV Emission Energy Stability Pulse-to-Pulse During $1 \mathrm{kHz}$ Burst.

\section{CONCLUSIONS AND FUTURE DEVELOPMENT PLANS}

From the data taken to date, we believe that the DPF is a viable candidate for an EUVL light source and that the SSPPM and power system design concept as described has proven to be capable of providing sufficient energy to the DPF load for EUV emission. The extremely low output inductance of this device allows for significant output currents necessary for proper DPF plasma "pinching". Experiments so far have demonstrated $2 \mathrm{kHz}$ burst operation and pulse-to-pulse charging regulation of $< \pm 0.25 \%$ towards the final goals of $5 \mathrm{kHz}$ and $\pm 0.1 \%$, respectively. Further development of the design will be necessary in order to reach the overall source power levels required for a production lithography tool. As a result, near term development plans include the addition of water cooling and other thermal management techniques, further improvement in pulse-to-pulse energy stability, continued exploration of DPF voltage scaling, and support of other EUV source development efforts. These other efforts will involve overall system efficiency improvement, collector and lithium delivery system development, pre-ionization system improvements, and overall thermal management.
In order to support these parallel efforts, an additional five DPF systems are currently being assembled at CYMER.

\section{ACKNOWLEDGEMENTS}

The authors would like to dedicate this paper to the late Dan Birx who worked with CYMER since 1990 and made immeasurable contributions towards the development of the overall EUV effort, as well as numerous SSPPM designs for excimer laser systems. His enthusiasm for solving problems was infectious and his example has made an immeasurable impact on many of us at CYMER. He lived his life with the same drive that he gave his work. Rare is the man with so much intelligence, diligence, capacity for joy, and respect for life. The world has lost a brilliant individual, and many of us lost a very close and dear friend. It was a pleasure and an honor to have known and worked with him, both as a technologist and as a fellow human being.

Igor Fomenkov and Terry Houston also deserve recognition and thanks for their support in generating some of the data provided in this paper and helping to set up many of the experiments on the DPF system.

\section{REFERENCES}

[1]G. Kubiak, V. Banine, and K. Ota, "Report from International EUVL Source Workshop", presented at the $2^{\text {nd }}$ International Workshop on Extreme Ultraviolet Lithography, San Francisco, CA, October 16-19, 2000.

[2] J.K. Ziemer, E.Y. Choueiri, and D. Birx, "Trends in Performance Improvements of a Coaxial Gas-Fed Pulsed Plasma Thruster", in Proc. IEPC-97-040, 1997.

[3] W.N. Partlo, I.V. Fomenkov, R. Oliver, and D.L. Birx, "Development of an EUV (13.5 nm) Light Source Employing a Dense Plasma Focus in Lithium Vapor", in Proc. SPIE, Vol. 3997, 2000, pp. 136-156.

[4] S. Lee, P. Lee, G. Zhang, X. Feng, V. Gribkov, M. Liu, A. Serban, and T. Wong, "High Rep Rate High Performance Plasma Focus as a Powerful Radiation Source", IEEE Trans. On Plasma Science, Vol. 26 \#4, pp. 1119-1126, Aug. 1998.

[5] M. Zakaullah, G. Murtaza, I. Ahmad, F.N. Beg, M.M. Beg, and M. Shabbir, "Comparative Study of Low Energy Mather-Type Plasma Focus Devices", Plasma Sources Sci. Technology, Vol. 4, pp. 117-124, 1995.

[6] W.N. Partlo, I.V. Fomenkov, R.M. Ness, R.I. Oliver, S.T. Melnychuck, and J.E. Rauch, "Progress Toward Use of a Dense Plasma Focus Device as a Light Source for Production EUV Lithography", Proc. SPIE, Vol. 4343-25, 2001. 